\title{
Reflectance confocal microscopy and optical coherence tomography for the diagnosis of bullous pemphigoid and pemphigus and surrounding sub-clinical lesions
}

Victor Desmond Mandel, ${ }^{1}$ Elisa Cinotti, ${ }^{2}$ Elisa Benati, ${ }^{1}$ Bruno Labeille, ${ }^{2}$ Silvana Ciardo, ${ }^{1}$ Cristina Vaschieri, ${ }^{1}$ Frédéric Cambazard, ${ }^{2}$ Jean-Luc Perrot, ${ }^{2}$ Giovanni Pellacani ${ }^{1}$

${ }^{1}$ Dermatology Unit; Department of Surgical, Medical, Dental and Morphological Sciences with Interest Transplant, Oncological and Regenerative Medicine; University of Modena and Reggio Emilia, Modena, Italy.

${ }^{2}$ Department of Dermatology; University Hospital of Saint-Étienne, Saint-Étienne, France.

Keywords: bullous pemphigoid; pemphigus; bullous disease; autoimmune disease; reflectance confocal microscopy; optical coherence tomography.

\begin{abstract}
Background: Diagnosis of bullous pemphigoid (BP) and pemphigus is based on clinical features, histology, immunofluorescence and laboratory data. ${ }^{1,2}$

Objectives: To evaluate features of BP and pemphigus at reflectance confocal microscopy (RCM) and optical coherence tomography (OCT) in order to provide a rapid non-invasive bed-side diagnosis. Secondary objective was to evaluate the detectability of clinically non-visible lesions.
\end{abstract}

Methods: This was an observational, retrospective, multicentre study in which patients with suspicious lesions for BP or pemphigus underwent clinical assessment, RCM, OCT, blood tests and skin biopsy for histological and direct immunofluorescence examinations from January 2014 to December 2015. A total of 72 lesions in 24 selected patients were evaluated. Additionally, apparently unaffected skin at two different distances [near $(1-2 \mathrm{~cm})$ and far $(2-3 \mathrm{~cm})$ ] from each lesion was examined in order to test sub-clinical lesion detectability. Instrument and acquisition procedures are described elsewhere. ${ }^{3,4}$ 
Results: RCM was able to detect sub-epidermal and intra-epidermal blisters respectively in $75 \%$ and $50 \%$ of the patients affected by BP and pemphigus. At OCT the exact blister level was identified in all patients. Acantholytic cells were observed only at RCM in pemphigus (62.5\%). Fibrin deposition inside the blisters was only found in BP, evidenced both at RCM and OCT. Among patients with BP, subclinical blisters were detected in $9(9.4 \%)$ clinically healthy skin, while among patients with pemphigus were observed in $10(20.8 \%)$ apparently unaffected skin.

Conclusions: RCM and/or OCT provide useful information for a rapid diagnosis of BP and pemphigus and for the identification of biopsy site. Combined use of RCM and OCT is optimal because associates the higher resolution of RCM with the greater penetration depth of OCT. OCT could be an optimal tool for treatment monitoring, especially in the cases of sub-clinical lesions.

\section{References}

1. Kneisel A, Hertl M. Autoimmune bullous skin diseases. Part 2: diagnosis and therapy. J Dtsch Dermatol Ges, 2011; 9: 927-47.

2. Feliciani C, Joly P, Jonkman MF, et al. Management of bullous pemphigoid: the European Dermatology Forum consensus in collaboration with the European Academy of Dermatology and Venereology. Br J Dermatol, 2015; 172: 867-77.

3. Samhaber KT, Buhl T, Brauns B, et al. Morphologic criteria of vesiculobullous skin disorders by in vivo reflectance confocal microscopy. J Dtsch Dermatol Ges, 2016; 14: 797-805.

4. Mogensen M, Morsy HA, Nurnberg BM, Jemec GB. Optical coherence tomography imaging of bullous diseases. J Eur Acad Dermatol Venereol, 2008; 22: 1458-64. 